\title{
Return of individual genomic research results: what do consent forms tell participants?
}

\author{
Stacey Pereira*, Jill Oliver Robinson and Amy L McGuire \\ Advances in genomic technology make possible the large-scale collection of genomic data for research purposes. Many \\ international initiatives seek to collect genomic data on large populations, often relying on existing collections to populate their \\ databases. As these efforts progress, the debate over whether or not to return individual genetic research results to study \\ participants remains an area of much contention. Some recommend returning results to participants only if the issue was \\ addressed in the original study consent form. Much of the data being used in current studies, however, may have been derived \\ from biospecimens collected years ago with consent documents that did not anticipate the possibility of returning individual \\ level genomic results. We conducted an analysis of informed consent documents from published genome-wide association \\ studies (GWAS) $(n=40)$ to explore whether future research use of biospecimens or data is anticipated, and if return of results is \\ addressed and how it is described to better understand participants' expectations for future disclosure. The majority $(70 \%)$ of \\ the GWAS consent documents we analyzed either stated explicitly that individual genomic results would not be returned or were \\ silent on the issue. This has implications for how researchers and members of Research Ethics Committees manage the return of \\ results from sequencing studies using legacy samples and data.
}

European Journal of Human Genetics (2016) 24, 1524-1529; doi:10.1038/ejhg.2016.76; published online 22 June 2016

\section{INTRODUCTION}

Advances in sequencing technology now make possible the large-scale collection of genomic data for research purposes. Many international initiatives seek to amass genomic data on large populations, often relying on existing collections to populate their databases (https:// genomicsandhealth.org/; http://h3africa.org/; http://www.uk10k.org/). As these efforts progress and the field of genomic science continues to make advances in human health, the debate over whether or not to return individual genetic research results, incidental, or secondary findings (hereinafter referred to collectively as 'research results') to study participants remains an area of much contention. Some argue that individual genetic research results should be routinely returned; ${ }^{1-4}$ others recommend that results be disclosed rarely or never. ${ }^{5-8}$ Studies suggest that participants generally want to receive individual results, ${ }^{9-13}$ and that other stakeholders, including Research Ethics Committee (REC) members and researchers, generally support the return of at least some research results. ${ }^{14-16}$ Researchers have also found support for returning findings from secondary analysis. ${ }^{17}$ Current guidelines endorse the return of results that are valid, clinically significant, and provide some measure of benefit to the participant. ${ }^{18-22}$ The American College of Medical Genetics and Genomics goes further and argues that there is an ethical and professional obligation to offer sequencing results related to 56 genes that meet their threshold of clinical significance. ${ }^{23}$

Most of these guidelines and recommendations, however, acknowledge that return of results needs to be addressed in the study consent form, and that the participant should have the opportunity to decide whether she would like to receive such results. ${ }^{19,24}$ In cases where researchers find individual research results that meet the threshold for return, but the consent form did not address the issue, recommendations suggest that researchers consult with their RECs to determine whether communication of the result is appropriate. In other cases where return of results was addressed in the consent, but did so by stating that results would not be returned, it may be difficult to justify communication of the result. ${ }^{25}$ Considering the widespread and increasing practice of using retrospectively collected biospecimens for secondary research and the increase in data sharing among genome researchers, this issue is of particular relevance now. Many of the samples being used in current studies were likely collected years ago using consent documents that were written before the existing guidelines and may not have accounted for the possibility of returning individual level genomic results, even though they may have specified that the biospecimens would be stored indefinitely and used in future research. We conducted an analysis of informed consent documents (ICDs) from published GWAS to explore whether future research is anticipated and if return of results is addressed, and how it is described to better understand participants' expectations for future disclosure.

\section{METHODS}

Study participants and procedures

ICDs from published GWAS were collected as part of a larger study investigating the practices and perspectives of GWAS investigators regarding the return of individual genetic research results. Potential participants were corresponding authors of articles identified using 'A Catalog of Published Genome-Wide Association Studies,' a list generated and maintained by the National Human Genome Research Institute. ${ }^{26}$ As of April 2010, there were 362 distinct corresponding authors for 517 published studies. Of these 362 authors, five were unreachable and one was deceased; the remaining 356 were invited by email to participate in an online survey of practices and perspectives on returning genetic research results to study participants. The invitation to participate 
included a description of the study, an assurance of confidentiality, and a link to the electronic survey. As approved by the Institutional Review Boards at Baylor College of Medicine and Harvard Medical School, consent to participate in the study was considered implicit if the investigator chose to follow the link to the survey. A total of 191 GWAS investigators completed the survey. The results of this survey are reported in a separate publication. ${ }^{27}$

At the end of the survey, participants were asked if they were willing to share a copy of the ICD associated with their GWAS by mail, fax, or email; if they indicated they did not have access to the ICD, they were asked for contact information for someone who could provide it. Of the 191 GWAS investigators who completed the survey, 50 agreed to share their ICD and 22 provided contact information for another individual. Those who agreed to share the consent and those individuals who were named as a contact for the ICD were contacted up to three times by email. Of these 72 individuals, three declined to send the consent and 22 were lost to follow-up. The remaining 47 individuals (13\% of corresponding authors) sent 93 documents from 53 studies.

\section{Data analysis}

We first evaluated the documents for inclusion in analysis and excluded documents that were not suitable for this study. We then analyzed ICDs for language about return of individual genetic research results. Two members of the research team (SP and JOR) read the ICDs and categorized them into one of four categories: (1) silent: the ICD has no language that refers to the return of individual genetic research results; (2) results returned: the ICD states that individual genetic research results will be returned to the participants; (3) no return of results: the ICD states that individual genetic research results will not be returned to the participants; and (4) possible to return results: the ICD contains language that states that return of results is possible (eg, under specific circumstances). We further classified the ICDs in this latter category to describe the instances in which it was possible to return results using three designations: (a) conditional: the ICD stated that results could be returned under certain circumstances, such as the discovery of an important health-related finding; (b) optional: the ICD (or an associated form) includes an option for participants to designate whether they would like to receive their individual genomic research results; (c) accessible: the ICD does not offer return of individual genomic research results, but specifies that participants can access their data. ICDs were characterized with more than one of these three designations when appropriate. We also analyzed the ICDs for language referring specifically to secondary research use of the biospecimens, as well as language about long-term biospecimen banking and creation of immortalized cell lines, both of which suggest the possibility of secondary research use. Interrater reliability was $95 \%$ and a consensus approach was used to resolve the discrepancies in categorization. Descriptive statistics of ICDs' language for return of individual genetic research results and language related to secondary use were explored.

\section{RESULTS}

We received 93 documents from 53 studies. After initially reviewing for suitability for inclusion in analysis, we excluded 44 documents (Figure 1), leaving 49 ICDs from 35 studies. Nine of the included studies had multiple ICDs. After analysis, we excluded an additional nine ICDs from studies that had multiple ICDs with identical return of results language. This left five studies for which two ICDs were included; in each case the different ICDs were for different populations or cohorts. Thus, 40 ICDs from 35 distinct GWAS were included for analysis. Twenty of the ICDs were from the United States and 20 were international. Thirty-two of the ICDs specified a year, which ranged from 1998 to 2010, with the median year being 2006 .

\section{Secondary research use}

Examples of ICD language used to describe plans for secondary research use, biospecimen banking, and the creation of cell lines are shown in Table 1. Of the 40 ICDs analyzed, 58\% $(n=23)$ referred

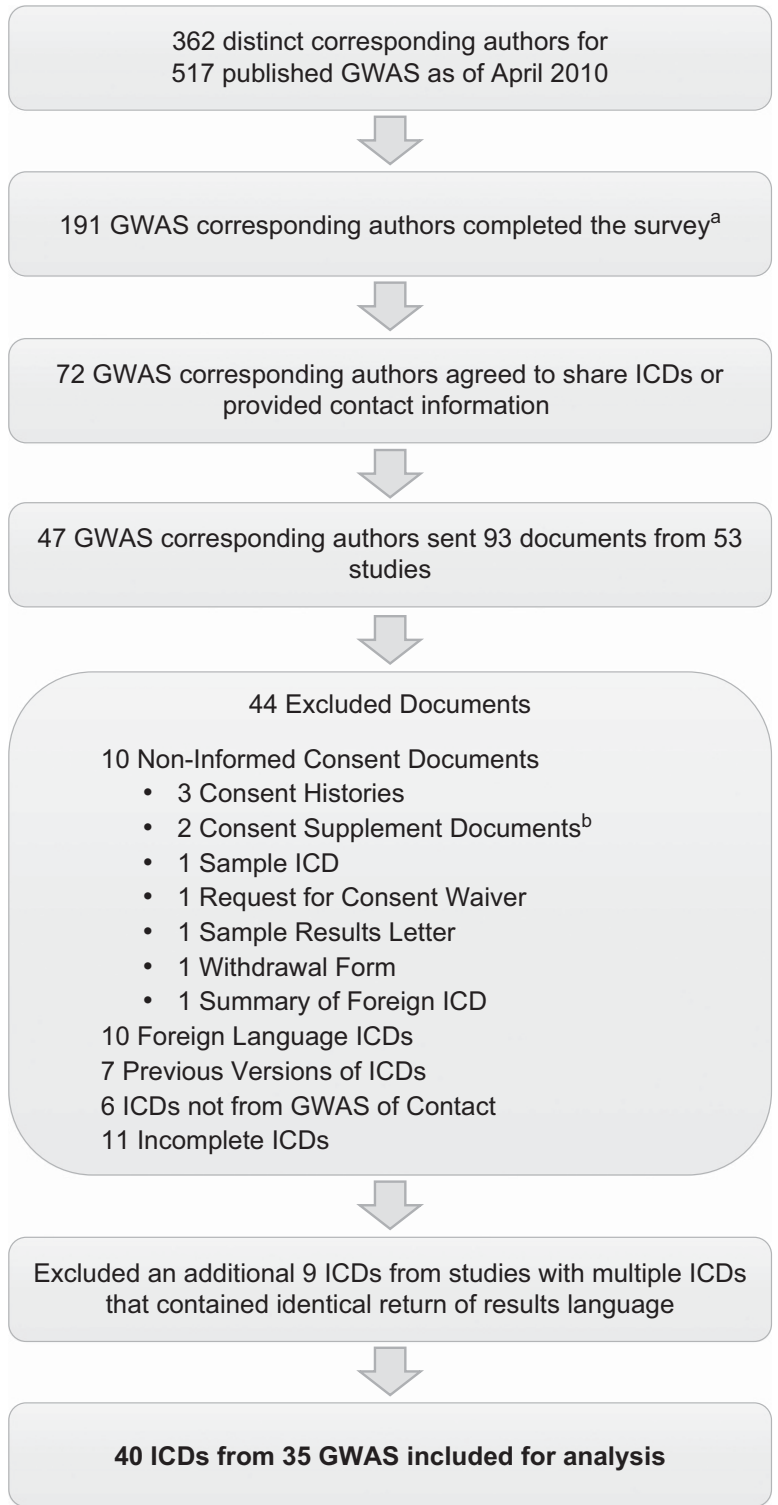

Figure 1 Flowchart depicting informed consent document collection and exclusions. aResults of the survey are reported in a separate publication ${ }^{27}$ ${ }^{b}$ Consent supplement documents consisted of an information sheet about informed consent and the previous study, and an information sheet on DNA.

specifically to secondary research use of the biospecimens beyond the GWAS described in the ICD. Other ICDs $(n=10)$ gave the participant the option to allow their biospecimen to be used for secondary research. Sixty-three percent $(n=25)$ of the ICDs stated that the biospecimen would be banked for an indefinite period of time, and one ICD gave the participant the option of allowing the biospecimen to be banked indefinitely. Three of the ICDs stated that the biospecimens would be banked for a specific period of time; two of these specified the biospecimen would be stored for 20 years, and the other for 50 years. Thirty percent $(n=12)$ of the ICDs stated that cell lines would be created from the biospecimen, whereas an additional two ICDs included an option to allow the creation of cell lines. Only one ICD stated that no secondary research would be done with the biospecimens without additional consent. 
Table 1 Examples of consent language related to future use and biospecimen storage

\begin{tabular}{|c|c|c|}
\hline \multirow[t]{2}{*}{ Issue } & \multicolumn{2}{|c|}{ Consent language } \\
\hline & Broad & Option/specific \\
\hline Secondary research use & $\begin{array}{l}\text { Your genetic material will be stored for the use in future studies on [disease } \\
\text { of GWAS] or other diseases. } \\
\text { At some time in the future [the biospecimen] will be used for tests to help in } \\
\text { research on [disease of GWAS] and conditions associated with [disease of } \\
\text { GWAS]. } \\
\text { Your participation in this study requires you to donate both blood and tissue } \\
\text { samples to be used for genetic testing for this and future [disease of GWAS] } \\
\text { studies. }\end{array}$ & $\begin{array}{l}\text { May we use the information, blood, urine and DNA obtained from you for } \\
\text { future studies of other disorders and diseases? Yes/no } \\
\text { I consent to: the information or samples that I provide being used for future } \\
\text { medical research into health, illness, and medical treatment. Yes/no }\end{array}$ \\
\hline Biospecimen banking & $\begin{array}{l}\text { The blood, cells, or genetic material (DNA) removed from you during the } \\
\text { course of this project will be maintained indefinitely at the [institution] for } \\
\text { research purposes or until the samples are gone. }\end{array}$ & $\begin{array}{l}\text { We would like to store the sample you have donated in a secure freezer for a } \\
\text { period of } 20 \text { years. }\end{array}$ \\
\hline & I understand that my sample may continue to be used indefinitely. & $\begin{array}{l}\text { The genetic material, blood samples, and cell lines will be stored by the } \\
\text { pharmaceutical products company that sponsors this study. The samples } \\
\text { will be kept for a period of } 50 \text { years, at which time they will be destroyed. }\end{array}$ \\
\hline & The sample and the information derived from it will be kept indefinitely. & \\
\hline Cell lines & $\begin{array}{l}\text { I/We understand that some of the blood cells collected may be treated so } \\
\text { that they survive in the laboratory for many years, and that DNA and RNA } \\
\text { can be extracted from them in the future. }\end{array}$ & $\begin{array}{l}\text { If a cell line has not already been collected, I agree to allow a cell line to be } \\
\text { made from a sample of my blood to provide a renewable supply of DNA. [A } \\
\text { cell line is a frozen sample of specially processed white cells from your } \\
\text { blood that allows us to grow more white cells and get more DNA from them } \\
\text { in the future as needed for the research projects]. Yes/no }\end{array}$ \\
\hline
\end{tabular}

In some cases, we will grow some of your white blood cells to maintain a continuous supply of DNA.

No additional research will be undertaken on these samples without your express consent.

Two of the 40 ICDs were silent on all three topics of secondary research use, long-term banking, and the creation of cell lines.

\section{Return of results}

Of the 40 ICDs from the 35 GWAS, none stated that genomic results would be returned unconditionally. Fifteen percent $(n=6)$ were silent, meaning that they did not contain any language pertaining to the return of individual level genomic results, and 55\% $(n=22)$ explicitly stated that individual level genetic results would not be returned to the participants. Some examples of this language include:

You will not be informed about any findings made using your genetic material (DNA).

Neither the results of the research on your chemical makeup, nor any other information about your genes can or will be made available to you or any other participant or your physicians.

Genetic analysis results from many individuals will be used to discover relationships between genes and [phenotype]. However, you should know that the genetic analysis performed on your blood will not be provided to you or your health care professional, and will not be used to treat any medical condition that you may have now or in the future.

The remaining 12 ICDs (30\%) were categorized as 'possible to return results.' As described above, the ICDs in this category did not state that results would be routinely returned, but rather included language that stated that genomic results could be returned. Five of these 12 ICDs were conditional, meaning that the ICD stated that results could be returned only if certain conditions were met. The conditions under which results could be returned included: finding results that were clinically relevant, finding results that were clinically relevant and being advised by an ethics committee to return them, and having 'important reasons' to return:

We would like to point out that all investigations are conducted for scientific purposes only and do not include comprehensive diagnosis of physical and neuropsychiatric diseases. Potential pathological changes (eg, in the blood) may therefore not be recognized. However, if in the event of any unexpected diagnostic findings that we consider important to your physical and mental health, we will inform you about them and discuss further action with you and, if necessary, with your attending doctor. If you are receiving clinical treatment at the [institution] at that time, we will also inform the doctor responsible for your care there.

As this is a research study, the significance of the results is unknown and the results will not affect the immediate care of you or your family. We will therefore not release individual results to you or your family, unless it clearly will influence you or your family's health and we are advised to release the information by the Ethics Committee.

Information on [nongenetic medical result] and the results of the genetic tests will only be given if there are important reasons to do so.

Six of the 12 ICDs were both conditional and optional, meaning that the ICD stated that genomic results could be returned only if certain conditions were met (as described above), and the participants had the option at the time of consent to indicate in the ICD or an accompanying document whether they would like to receive such results:

I_IYES I__ INO If a genetic condition is identified that may have potentially important health and treatment implications for me, I 
agree to allow the [research group] to notify me and with my permission to notify my physician.

However, it is possible that we find that you carry a gene that causes a high risk of [disease]. This may have implications for your family because it may indicate that members of your family might have an increased risk of developing [disease] or other diseases such as [disease]. When you give the sample, we will provide you with a form where you can indicate whether or not you would like to be informed if we find something that is of importance for your health or that of your family. If you do wish to be informed, we will arrange for you to see a specialist doctor in genetics to discuss what the result might mean for you and your family.

I'm aware of the fact that the present research does not have a diagnostic goal and that I will not receive any specific information on the results of this project. Nevertheless, if in the future development of the research were to emerge exceptionally relevant information for my health status and my life:

I__ II want to be informed I__ II want that my doctor be informed ___I don't want to be informed.

The remaining ICD did not offer results to the participants, but explained that the genetic data were accessible to them:

If you wish, you will be given access for inspection of your genetic data at the [institution] upon completion of the genetic analysis. This data are meant for research purposes only. Except in very rare cases only, such data do not provide any information that might be of medical relevance for personal diagnoses or treatment at this point in time. It must be assumed that these scientific research data are only incomplete and that it may become outdated in the near future in terms of the current state of the research (emphasis in original).

\section{Within study variation}

We included multiple ICDs for analysis from five studies that had separate ICDs for different populations or cohorts and contained different language regarding return of results. For one study, we received seven separate ICDs that were written for different family members (child, parent, and sibling) and different ages (under 12, 12-15, and 16 and older). We included only two of these ICDs because there were only two variations of return of results language across them in the overall analysis, but we discuss all seven in this section to explore the intra-study variation in these ICDs. Of the seven ICDs from this one study, we found that four ICDs were for participants under 16 and were each silent regarding the return of individual genomic results, whereas the three ICDs for participants 16 and older, each stated that results would not be returned.

For another study we received two ICDs, each for the population of a different geographical region. In this study, one ICD stated that results would not be returned, and the other was silent. In two other studies, we received two ICDs each: one for an affected population and one for a general population. These studies took different approaches regarding return of results to their different populations: in one study, the ICD for affected participants was silent, whereas the ICD for the general population included language that was both conditional and optional. The other study had the reverse approach, with the affected population ICD containing language that was conditional and optional, and the ICD for the general population containing no language referring to the return of results. In the last study that sent multiple ICDs, there were different ICDs for different generations of participants (in terms of when they enrolled in the study). The ICD for the earlier generation contained return of results language that was both conditional and optional, and the ICD for the later generation stated that no results would be returned. Intra-study variation in the ICDs from these five studies could be due to them being written at different times; however, we did not have reliable information on when and by whom the consents were drafted, and therefore could not make that determination.

\section{DISCUSSION}

Though many of these original GWAS were unlikely to yield clinically significant and actionable results due to the nature of those early studies, the majority of the ICDs $(83 \%, n=33)$ either directly stated that the biospecimens would be used in secondary research, or gave the participant an option to allow secondary research. Of the seven remaining ICDs, five included language about, or an option for, long-term storage and/or creation of immortalized cell lines from the biospecimens, both of which suggest that the biospecimens might be used in secondary research (though one ICD that allowed for indefinite storage stated that the biospecimen would not be used in secondary research). Secondary and ongoing use, and sharing of the biospecimens and data increases the likelihood of discovering an individual genetic research result that meets the threshold for return.

The majority (70\%) of the GWAS ICDs analyzed, however, either stated explicitly that individual genomic results would not be returned or were silent on the issue. This is not surprising, as many of these ICDs were developed prior to the current guidelines, ${ }^{19}$ and other studies have found similar results. ${ }^{28}$ Yet, our research with GWAS investigators suggests that, although they believe results should be returned in some circumstances, the decision about whether to communicate them to participants is heavily influenced by what was written in the original ICD, and that investigators may view the ICD similar to a contract and feel bound to its terms. ${ }^{29}$ RECs, likewise, are expected to ensure that return of results is consistent with the original ICD (https://gds.nih.gov/ 13faqs_gds.html). This raises important questions about what investigators ought to do if they discover a result they feel compelled to disclose, but the ICD does not address or even prohibits results being returned.

The ICDs we reviewed that did allow for return of some individual results $(n=12)$ were generally not specific about what types of results could be returned (ie, results from the original research study, secondary research, or incidental findings), aside from two ICDs that described incidental findings. According to other research, this lack of clarity is not uncommon even in more recently written ICDs. ${ }^{30}$ Thus, even when the ICD leaves open the option for returning individual genetic results, investigators may feel conflicted about returning a result that was unrelated to the original study described in the ICD, and therefore not anticipated by the participant.

Whether results from secondary analysis conducted with previously collected biospecimens can be returned to participants is also contingent on whether it is possible to recontact the participants. If the biospecimens are not stored in such a way that participant information is accessible to the investigator, there is no possibility of returning the result. Even if contact information is available, whether 
the participant can be contacted is also influenced by the original ICD. If recontact of the participant is not permitted by the ICD, it may be more difficult to justify communication of any secondary results. In our sample, 25 of the ICDs did mention the possibility of recontact, though this was most often in the context of recontact to offer participation in additional research or to collect additional information and/or biospecimens. Where recontact is possible and permissible by the original ICD, it may be justifiable to return an important finding to the participant. However, this raises additional questions about the availability of resources to cover the costs of validating the finding and contacting the participants, and how the communication should be handled and by whom.

This issue may be less relevant in the future, as we move from onetime consent and sample collection toward models of research participation that require ongoing and long-term participant engagement, and may allow for open communication between participants and researchers. A key feature of the Precision Medicine Initiative (https://www. whitehouse.gov/the-press-office/2015/01/30/fact-sheet-president-obamas-precision-medicine-initiative; https://www.congress.gov/bill/114th-congress/house-bill/6/text), for instance, is the creation of a cohort of one million or more volunteers who will be asked to provide biospecimens and health records, as well as ongoing health data related to lifestyle and environmental exposure information. The ongoing engagement of such a cohort creates a stronger relationship between the participants and the researchers; in such a context, return of important research results may be more feasible and appropriate.

This study has several limitations. The ICDs that we analyzed were used to collect samples for GWAS that were published as of 2010 . Though most of the ICDs specified a date, it was not always clear what that date reflected (eg, original approval date and most recent approval date), and we are unable to determine the range of time that ICDs may have been in use; many may have been used to collect samples for years leading up to the GWAS and may also have been in use since. In addition, because the ICDs generally did not distinguish between different types of results (eg, individual results from the initial research study, secondary results, incidental findings, etc), we are unable to analyze the ICDs' language on return of results with regard to result type or actionability of potential findings. Finally, due to our sample size of 40 ICDs and the necessity of excluding foreign language documents, our analysis may not be representative of all ICDs that were in use during this time. Future research should focus on whether there has been improvement in ICD language over the last 6 years, as there are now guidelines in place that recommend the return of some results. These guidelines, however, offer little in the way of specific guidance on how to address this issue, and our experience has been that there continues to be great variability in how return of results is addressed in genomic research ICDs.

Ultimately, the decision of whether to return individual genetic research results to participants, when the ICD does not permit such return, warrants careful consideration on a case-by-case basis. Investigators and REC members may want to consider the context and original intention of the study. ${ }^{29}$ For instance, in cases where the purpose of the original research was to find a diagnosis for a participant, there may be greater obligation to return certain results, even if the ICD did not address this issue. The nature of the relationship between the researcher and the participants should also be considered. In situations where there is an existing and continued relationship, such as when researchers partner with disease advocacy organizations, there again there may be greater responsibility to return meaningful results to participants. Considering this question on a case-by-case basis allows investigators and REC members to make decisions consistent with the ethical principles of transparency, trust, and respect.

\section{CONFLICT OF INTEREST}

The authors declare no conflict of interest.

\section{ACKNOWLEDGEMENTS}

This work was funded by support from the Baylor College of Medicine Clinical and Translational Research Program and the Baylor Annual Fund. ALM was supported by National Institutes of Health grant R21HG006612. We would like to thank Drs Steven Joffe, Rachel Ramoni, and Sharon Plon for their valuable contributions to this project. In addition, we thank Hayley Peoples for her research assistance on this paper.

1 Fernandez C: Public expectations for return of results-time to stop being paternalistic? Am J Bioeth 2008; 8: 46-48.

2 Fernandez CV, Kodish E, Weijer C: Informing study participants of research results: an ethical imperative. IRB 2003; 25: 12-19.

3 Shalowitz DI, Miller FG: Disclosing individual results of clinical research: implications of respect for participants. JAMA 2005; 294: 737-740.

4 Sharp RR, Foster MW: Clinical utility and full disclosure of genetic results to research participants. Am J Bioeth 2006; 6: 42-44.

5 Clayton EW, Ross LF: Implications of disclosing individual results of clinical research. JAMA 2006; 295: 37.

6 Meltzer LA: Undesirable implications of disclosing individual genetic results to research participants. Am J Bioeth 2006; 6: 28-30.

7 Miller FA, Christensen R, Giacomini M, Robert JS: Duty to disclose what? Querying the putative obligation to return research results to participants. J Med Ethics 2008; 34: 210-213.

8 Clayton EW, McGuire AL: The legal risks of returning results of genomics research. Genet Med 2012; 14: 473-477.

9 Kaufman D, Murphy J, Scott J, Hudson K: Subjects matter: a survey of public opinions about a large genetic cohort study. Genet Med 2008; 10: 831-839.

10 Murphy J, Scott J, Kaufman D, Geller G, LeRoy L, Hudson K: Public expectations for return of results from large-cohort genetic research. Am J Bioeth 2008; 8: 36-43.

11 Shalowitz DI, Miller FG: The search for clarity in communicating research results to study participants. J Med Ethics 2008; 34: e17.

12 Wendler D, Emanuel $\mathrm{E}$ : The debate over research on stored biological samples: what do sources think? Arch Intern Med 2002; 162: 1457-1462.

13 Bollinger JM, Scott J, Dvoskin R, Kaufman D: Public preferences regarding the return of individual genetic research results: findings from a qualitative focus group study. Genet Med 2012; 14: 451-457.

14 Beskow LM, O'Rourke PP: Return of genetic research results to participants and families: IRB perspectives and roles. J Law Med Ethics 2015; 43: 502-513.

15 Gliwa C, Yurkiewicz IR, Lehmann LS, Hull SC, Jones N, Berkman BE: Institutional review board perspectives on obligations to disclose genetic incidental findings to research participants. Genet Med 2015; e-pub ahead of print 19 November 2015; doi:10.1038/gim.2015.149.

16 Klitzman R, Appelbaum PS, Fyer A et al: Researchers' views on return of incidental genomic research results: qualitative and quantitative findings. Genet Med 2013; 15: 888-895.

17 Middleton A, Morley KI, Bragin E et al: Attitudes of nearly 7000 health professionals, genomic researchers and publics toward the return of incidental results from sequencing research. Eur J Hum Genet 2016; 24: 21-29.

18 Bookman EB, Langehorne AA, Eckfeldt JH et al: Reporting genetic results in research studies: summary and recommendations of an NHLBI working group. Am J Med Genet A 2006; 140: 1033-1040.

19 Fabsitz RR, McGuire A, Sharp RR et al: Ethical and practical guidelines for reporting genetic research results to study participants: updated guidelines from a National Heart, Lung, and Blood Institute working group. Circ Cardiovasc Genet 2010; 3: 574-580.

20 Knoppers BM, Joly Y, Simard J, Durocher F: The emergence of an ethical duty to disclose genetic research results: international perspectives. Eur J Hum Genet 2006; 14: $1170-1178$.

21 National Bioethics Advisory Commission. Research Involving Human Biological Materials: Ethical Issues and Policy Guidance. Rockville, MD, USA: NBAC, 1999.

22 Jarvik GP, Amendola LM, Berg JS et al: Return of genomic results to research participants: the floor, the ceiling, and the choices in between. Am J Hum Genet 2014; 94: 818-826.

23 Green RC, Berg JS, Grody WW et al: ACMG recommendations for reporting of incidental findings in clinical exome and genome sequencing. Genet Med 2013; 15: 565-574.

24 McGuire AL, Beskow LM: Informed consent in genomics and genetic research. Annu Rev Genomics Hum Genet 2010; 11: 361-381. 
25 Wolf SM, Crock BN, Van Ness B et al: Managing incidental findings and research results in genomic research involving biobanks and archived data sets. Genet Med 2012; 14: 361-384.

26 Hindorff LA, Junkins HA, Manolio TA. A catalog of published genome-wide association studies. 2013. http://www.genome.gov/26525384 (accessed 22 February 2013).

27 Ramoni RB, McGuire AL, Robinson JO, Morley DS, Plon SE, Joffe S: Experiences and attitudes of genome investigators regarding return of individual genetic test results. Genet Med 2013; 15: 882-887.
28 Munung NS, Marshall P, Campbell M et al: Obtaining informed consent for genomics research in Africa: analysis of H3Africa consent documents. J Med Ethics 2016; 42. 132-137.

29 McGuire AL, Robinson JO, Ramoni RB, Morley DS, Joffe S, Plon SE: Returning genetic research results: study type matters. Per Med 2013; 10: 27-34.

30 Henderson GE, Wolf SM, Kuczynski KJ et al: The challenge of informed consent and return of results in translational genomics: empirical analysis and recommendations. J Law Med Ethics 2014; 42: 344-355. 